\title{
Do Pregnant Women Consume Enough Creatine? Evidence from NHANES 2011-2018
}

\author{
Sergej M. Ostojic ${ }^{a}$ Scott C. Forbes ${ }^{b}$ Darren G. Candow ${ }^{c}$

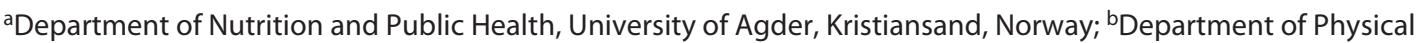 \\ Education Studies, Faculty of Education, Brandon University, Brandon, MB, Canada; 'Faculty of Kinesiology and \\ Health Studies, University of Regina, Regina, SK, Canada
}

Dear Editor,

Creatine has been proposed as a conditionally essential nutrient primarily due to its role in various energy-demanding processes across the human body [1], including reproduction and pregnancy. Preliminary data show an increased demand for maternal creatine due to the rapid growth and increased metabolic requirements of the fetus, with low creatine availability associated with poor pregnancy outcomes (for a detailed review, see [2]). Approximately $50 \%$ of daily creatine needs are accomplished through an omnivorous diet, and the required intake in healthy women ingesting a typical Western-pattern diet is estimated to be $\sim 0.75 \mathrm{~g}$ per day [3]. Whether pregnant women manage to consume sufficient dietary creatine remains unknown. Here, we determined the daily amount of creatine consumed in a typical diet in US pregnant women using cumulative data from the National Health and Nutrition Examination Survey (NHANES) 20112012, 2013-2014, 2015-2016, and 2017-2018 rounds.

For this brief report, we isolated data from 4 NHANES databases for all female respondents aged 20-44 years who provided information regarding dietary intake and pregnancy status (yes/no) at the time of screening $(2,545$ women, aged $33.7 \pm 6.8$ years). The final sample consisted of 173 pregnant and 2,372 nonpregnant women. A daily intake of creatine was calculated using the Dietary Data

karger@karger.com
www.karger.com/anm
Karger $\%$
BOPEN ACcEss

(C) 2021 The Author(s)

Published by S. Karger AG, Basel

This article is licensed under the Creative Commons Attribution 4.0 International License (CC BY) (http://www.karger.com/Services/ OpenAccessLicense). Usage, derivative works and distribution are permitted provided that proper credit is given to the author and the original publisher. databases on individual foods collected through in-person recall interviews, using the average amount of creatine $(3.88 \mathrm{~g} / \mathrm{kg})$ across all creatine-rich food sources [4]. The ethical approval to conduct the NHANES rounds was granted by the US National Center for Health Statistics Research Ethics Review Board (Protocol No. 2011-17, Continuation of Protocol No. 2011-17, Protocol No. 2018-01), and informed consent was obtained from all respondents.

The mean dietary intake of creatine across the entire sample was $0.76 \pm 0.77 \mathrm{~g}$ per day ( $95 \%$ confidence interval [CI], from 0.73 to $0.79 \mathrm{~g} /$ day). The pregnant women consumed $0.83 \pm 1.07 \mathrm{~g}$ of creatine per day $(95 \% \mathrm{CI}$, from 0.66 to 0.98 ; median $0.66 \mathrm{~g} /$ day; $5 \%$ trimmed mean $0.67 \mathrm{~g} /$ day) (Fig. 1). Nonpregnant women ingested $0.75 \pm 0.74 \mathrm{~g}$ of creatine daily (95\% CI, 0.72-0.78), and no differences were found for daily creatine intake between pregnant and nonpregnant women $(p=0.82)$. In addition, $57.2 \%$ of pregnant women (99 out of 173 respondents) consumed creatine below $0.75 \mathrm{~g}$ per day, with $17.3 \%$ (30 out of 173) consuming no creatine at all. The highest intake across the pregnant women subsample was reported in a female aged 33 years consuming $9.32 \mathrm{~g}$ of creatine per day.

Our study was the first that reported daily intake of creatine from a regular diet in pregnant women. Although
Correspondence to:

Sergej M. Ostojic, sergej.ostojic@ uia.no 


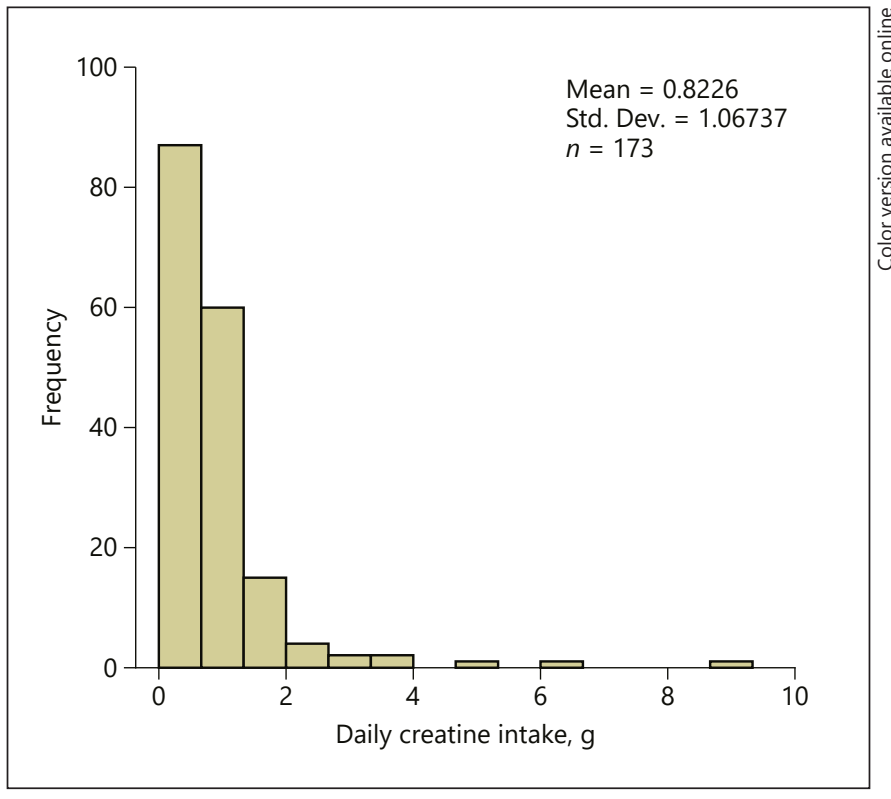

Fig. 1. Histogram of daily creatine intake in pregnant women $(n=$ 173).

a high variability of creatine intake has been demonstrated in our report, it appears that on average, pregnant women ingest adequate amounts of creatine in a typical diet. The mean creatine intake of $0.83 \mathrm{~g}$ per day for pregnant women is $\sim 11 \%$ above the estimated dietary creatine requirements. However, approximately 6 out of 10 pregnant women (57.2\%) consumed creatine below the recommended amounts for an adult female, suggesting a possible risk of creatine malnutrition in this population. This is of concern since an impaired turnover of maternal creatine during pregnancy may compromise normal energy metabolism, impede fetal growth, and provoke unfavorable perinatal outcomes [5-7]. Furthermore, the magnitude of creatine deficiency may be even greater than reported, since the requirements for dietary creatine in pregnancy (although presently unknown) might be well over $0.75 \mathrm{~g}$ per day. A total daily turnover of creatine should also include endogenously synthesized creatine that normally provides a half of daily creatine needs [1]; no information is currently available whether internal synthesis of creatine is altered in pregnant women. Additional studies are urgently warranted to discern creatine needs in pregnancy and examine possible links between low creatine intake and pregnancy outcomes and rigorous randomized controlled trials to determine the efficacy of alterations in dietary creatine in pregnant women below the requirements.

\section{Statement of Ethics}

The study was conducted according to the guidelines of the Declaration of Helsinki. The ethical approval to conduct the NHANES rounds was granted by the US National Center for Health Statistics Research Ethics Review Board (Protocol No. 2011-17, Continuation of Protocol No. 2011-17, Protocol No. 2018-01), and written informed consent was obtained from all respondents.

\section{Conflict of Interest Statement}

S.M.O. serves as a member of the Scientific Advisory Board on creatine in health and medicine (AlzChem LLC). S.M.O. owns patent "Sports Supplements Based on Liquid Creatine" at European Patent Office (WO2019150323 A1) and active patent application "Synergistic Creatine" at UK Intellectual Property Office (GB2012773.4). S.M.O. has served as a speaker at Abbott Nutrition, a consultant of Allied Beverages Adriatic and IMLEK, and an advisory board member for the University of Novi Sad School of Medicine and has received research funding related to creatine from the Serbian Ministry of Education, Science, and Technological Development, Provincial Secretariat for Higher Education and Scientific Research, AlzChem GmbH, KW Pfannenschmidt $\mathrm{GmbH}$, ThermoLife International LLC, and Monster Energy Company. S.M.O. does not own stocks and shares in any organization. S.C.F. has previously served as a scientific advisor for a company that sold creatine. D.G.C. has conducted industry-sponsored research involving creatine supplementation and received creatine donations for scientific studies and travel support for presentations involving creatine supplementation at scientific conferences. In addition, D.G.C. serves on the Scientific Advisory Board for AlzChem (a company that manufactures creatine). The funders had no role in the design of the study; in the collection, analyses, or interpretation of data; in the writing of the manuscript; or in the decision to publish the results.

\section{Funding Sources}

This work was not funded by any agency in the public, commercial, or not-for-profit sectors.

\section{Author Contributions}

The authors' responsibilities were as follows: S.M.O. designed and wrote the draft of the manuscript and has primary responsibility for the final content, and all authors read and approved the final version of the manuscript.

\section{Data Availability Statement}

Data described in the manuscript will be made available upon request. 


\section{References}

1 Ostojic SM, Forbes SC. Perspective: creatine, a conditionally essential nutrient: building the case. Adv Nutr. 2021:nmab111. (in press).

2 Muccini AM, Tran NT, de Guingand DL, Philip M, Della Gatta PA, Galinsky R, et al. Creatine metabolism in female reproduction, pregnancy and newborn health. Nutrients. 2021;13(2):490.

3 Brosnan JT, Brosnan ME. Creatine: endogenous metabolite, dietary, and therapeutic supplement. Annu Rev Nutr. 2007;27:24161.
4 Bakian AV, Huber RS, Scholl L, Renshaw PF, Kondo D. Dietary creatine intake and depression risk among U.S. adults. Transl Psychiatry. 2020;10(1):52.

5 Heazell AE, Bernatavicius G, Warrander L, Brown MC, Dunn WB. A metabolomic approach identifies differences in maternal serum in third trimester pregnancies that end in poor perinatal outcome. Reprod Sci. 2012; 19(8):863-75.
6 Dickinson H, Davies-Tuck M, Ellery SJ, Grieger JA, Wallace EM, Snow RJ, et al. Maternal creatine in pregnancy: a retrospective cohort study. BJOG. 2016;123(11): 1830-8.

7 Alessandrì MG, Strigini F, Cioni G, Battini R. Increased creatine demand during pregnancy in Arginine: glycine amidino-transferase deficiency: a case report. BMC Pregnancy Childbirth. 2020;20(1):506. 